\title{
Visualization of Multi-Scale Structures for Different Initial Conditions of Turbulent Wake Using Wavelets
}

\author{
Hui LI ${ }^{\mathbf{o}}$ (Kagoshima University), Yu ZHOU (The Hong Kong Polytechnic University) \\ Masahiro TAKEI (Nihon University), Yoshifuru SAITO (Hosei University) \\ Kiyoshi HORII (Shirayuri Women College)
}

\begin{abstract}
The turbulent structures of various scales behind the solid and porous wake-generating bodies with the same characteristic dimension have been studies by the wavelet vector multi-resolution technique. Making a comparison of instantaneous sectional streamlines of various scales obtained with each wake generator, it was found that the behavior of large- and intermediate-scale structures in wakes depends on the initial conditions and the small-scale structures is independent of the initial conditions. The most significant contribution to Reynolds stress and turbulent energy appears to come from the large-scale and rib-like structures in the cylinder wake. In the screen wake, however, only the large-scale structure exhibits more contribution.
\end{abstract}

Keywords: Vortex, Turbulent Wake, Vector Wavelet Multi-resolution Analysis, Reynolds Stress

\section{Introduction}

It is well-known fact that the characteristics of the turbulent structure in the wake may differ as the wake-generating bodies or initial conditions differ. A number of investigators (Screenivasan, 1981; Zhou and Antonia, 1994) have studied the effect of the initial conditions on the behavior of a near turbulent wake. However, most of these investigations focus on organized structures, i.e. averaged large-scale structures, and clarify their contribution to turbulent transfer process behind various generators. It is difficult to deduce intermediate- and small-scale structures using the conventional techniques. As a result, until now there is a lack of information on the turbulent structures of various scales for different initial conditions. In the past decade, there has been a growing interest in the use of wavelet analysis for turbulent flow data $(\mathrm{Li}, 1998)$. This technique allows time- and frequency-space analyses to be combined and produces a potentially clearer picture of time-frequency localization of turbulent structures. $\mathrm{Li}$ et al. (1999) applied the two-dimensional orthogonal wavelets to turbulent images for extracting the multi-resolution turbulent structures, however, few investigations concerned the extraction of turbulent structures in terms of time and scale from the measured velocity vector field. The aim of the present work is to apply the vector wavelet multi-resolution analysis to the experimental velocity vector data for examining the turbulent structures of various scales behind different wake-generating bodies. The experimental methodology that is used consist in first extracting the turbulent structures of various scales by vector wavelet multi-resolution technique. Then the velocity vector field is decomposed in both Fourier and physical spaces, thus allowing the comparison of instantaneous sectional streamlines of various scales obtained with each wake generator. The contributions the various scale motions make to the Reynolds stress have been quantified in each case.

\section{Vector Wavelet Multi-resolution Analysis}

Let us consider a two-dimensional vector field $\vec{f}\left(x_{1}, x_{2}\right)$ and a two-dimensional orthogonal wavelet basis

$$
\Psi_{m,, n_{1} ; m_{2}, n_{2}}\left(x_{1}, x_{2}\right)=2^{-\left(m_{1}+m_{2}\right) / 2} \psi\left(2^{-m_{1}} x_{1}-n_{1}\right) \psi\left(2^{-m_{2}} x_{2}-n_{2}\right)
$$

where $\psi(x)$ is an one-dimensional orthogonal wavelet basis.

The two-dimensional discrete vector wavelet transform is defined as

$$
\overrightarrow{W f}_{m_{1}, n_{1} ; m_{2}, n_{2}}=\int_{-\infty}^{\infty} \int_{-\infty}^{\infty} \bar{f}\left(x_{1}, x_{2}\right) \Psi_{m_{1}, n_{1} ; m_{2}, n_{2}}\left(x_{1}, x_{2}\right) d x_{1} d x_{2}
$$

The reconstruction of the original vector field can be achieved by using

$$
\vec{f}\left(x_{1}, x_{2}\right)=\sum_{m_{1}} \sum_{m_{2}} \sum_{n_{1}} \sum_{n_{2}} \overrightarrow{W f}_{m_{1}, n_{1} ; m_{2}, n_{2}} \Psi_{m_{1}, n_{1} ; m_{2}, n_{2}}\left(x_{1}, x_{2}\right)
$$


The procedure of the wavelet vector multi-resolution analysis can be summarized in two steps:

(1) Wavelet coefficients or wavelet spectrum of vector data is computed based on the discrete vector wavelet transform of Eq. (2).

(2) Inverse vector wavelet transform of Eq. (3) is applied to wavelet coefficients at each wavelet level, and vector components are obtained at each level or scale or in the wavelet spaces.

It is evident that a sum of all vector components in the wavelet spaces can reconstruct the original vector. The wavelet vector multi-resolution analysis may process fewer data by selecting the relevant details that are necessary to perform an extraction of the multi-scale structures and coherent structures, and decompose the vector data in both Fourier and physical spaces. The technique is unique in terms of its capability to separate turbulence structures of different scales. In this paper, we use the Daubechies family with index $N=20$, which is not only orthogonal, but also has smoothness and compact support, as the orthogonal wavelet basis.

\section{Experimental Setup}

Experiments were carried out in a low turbulence wind tunnel with a $2.4 \mathrm{~m}$ long working section $(0.35 \mathrm{~m}$ x $0.35 \mathrm{~m}$ ). A circular cylinder and a screen of $50 \%$ solidity with the same height $(h=12.5 \mathrm{~mm})$ were used and installed in the mid-plane and spanned the full width of the working section. Two orthogonal arrays (Fig.1), each of eight X-wires, were used. Eight $\mathrm{X}$-wires were aligned in the $(x, y)$-plane, i.e. the plane of mean shear, and eight in the $(x, z)$-plane, which is parallel to both the cylinder axis and the streamwise direction. The sixteen $\mathrm{X}$-wires allow velocity fluctuations $u$ and $v$ in the $(x, y)$-plane and $u$ and $w$ in the $(x, z)$-plane to be obtained simultaneously with a sampling frequency of $f_{s}=3.5 \mathrm{kHz}$. The nominal spacing between $X$-wires was about $5 \mathrm{~mm}$. Measurements were made at $x / h=20$ and a constant free stream velocity $\left(U_{\infty}=6.7 \mathrm{~m} / \mathrm{s}\right)$. The corresponding Reynolds number $R e$ was 5600.

\section{Instantaneous Turbulent Structures}

In order to gain insight into the multi-scale turbulent structures, we apply wavelet vector multi-resolution analysis to the experimental velocity vector data of turbulent wakes.

The spanwise and lateral components of vorticity at each of the scales can be also defined in terms of the derivatives of the instantaneous velocity components $U_{i}=\bar{U}+u_{i}$ and $V_{i} \cong v_{i}(\bar{V} \cong 0)$, where an overbar

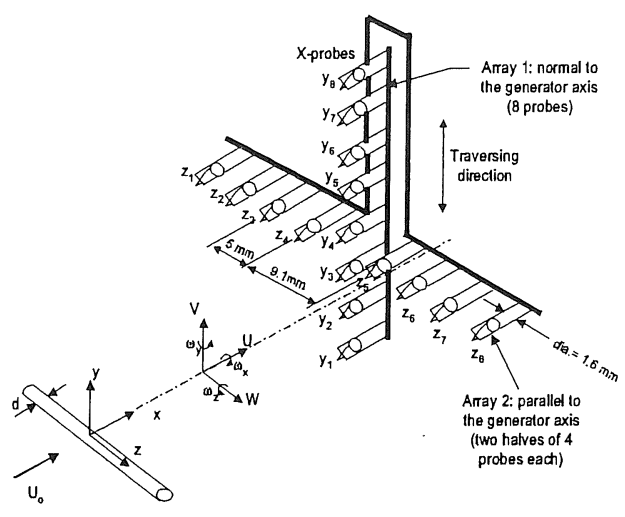

Figure 1: Experimental arrangement.

denotes time averaging and the index, $i$, stands for scale, respectively, viz.

$$
\omega_{z i}=\frac{\partial V_{i}}{\partial x}-\frac{\partial U_{i}}{\partial y}=\frac{\partial v_{i}}{\partial x}-\frac{\partial\left(\bar{U}+u_{i}\right)}{\partial y} \approx \frac{\Delta v_{i}}{\Delta x}-\frac{\Delta\left(\bar{U}+u_{i}\right)}{\Delta y}
$$

In above equations, $\Delta y \approx 5.0 \mathrm{~mm}$ is spacing between two X-wires; $\Delta x=-U_{c} \Delta t$, where $\Delta t=1 / f_{s}$ and $U_{c}=$ $0.87 U_{\infty}$ (Zhou \& Antonia 1992) is the average convection velocity.

Figure 2 shows the measured sectional streamlines in the cylinder and screen wakes in the $(x, y)$-plane. The flow direction is right to left. It is suggested that the cylinder and screen wakes have different geometrical characteristics of the vortex structure, e.g., shape, size, and spacing of the vortices. However, only the large-scale structures can be identified. However, it is difficult to identify the structures smaller than large-scale vortices in the measured streamlines (Fig.2) for both the cylinder and screen wakes. The measured velocity vector data were decomposed based on the wavelet vector multi-resolution analysis. Figures 3 and 4 show the decomposed sectional streamlines of the cylinder and screen wakes, which were calculated from the components of velocity vector for wavelet levels 9 to 12 in the $(x, y)$-plane. The central frequencies are 109 , 219,438 and $875 \mathrm{~Hz}$ for the cylinder wake and 130, 260 and $510 \mathrm{~Hz}$ for the screen wake, representing different scales of turbulence structures. As shown in Fig. 3 (a), six vortical structures are easily extracted in the cylinder wake. These structures correspond quite well to the large-scale structures in Fig.2 (a), which occur at frequencies around the average frequency (108 $\mathrm{Hz}$ ) of Karman vorticies. Figure 4(a) exhibits ten vortex pairs in the screen wake with the central frequency of $130 \mathrm{~Hz}$. These structures also correspond quite well to the large-scale structures in Fig.2 (b), and their occurrence is rather periodical. They are apparently the uppermost and energy-containing structures. Making a comparison between the cylinder and screen wakes, the size of the large-scale structure in cylinder wake is larger than that in screen wake, and the vortices of 


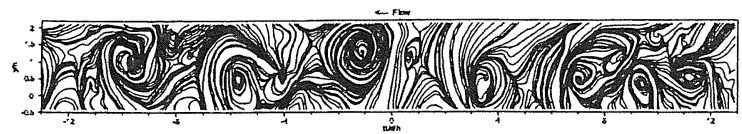

Figure 2(a): Measured cylinder wake.

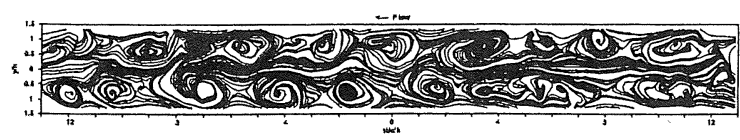

Figure 2(b): Measured screen wake.

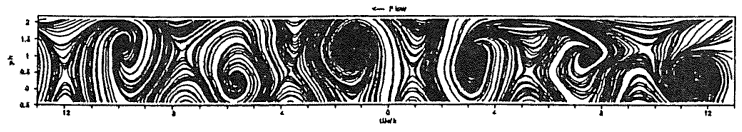

(a) $f=109 \mathrm{~Hz}$

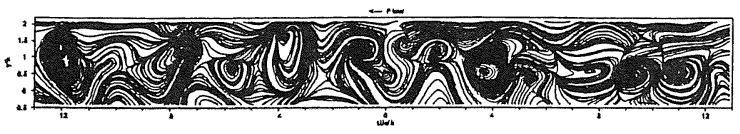

(b) $f=219 \mathrm{~Hz}$

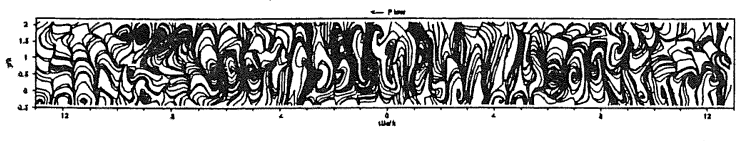

(c) $f=438 \mathrm{~Hz}$

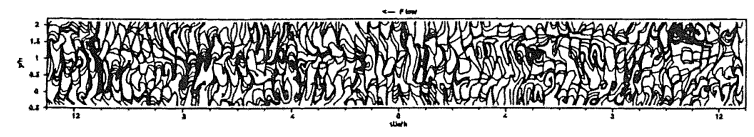

(d) $f=875 \mathrm{~Hz}$

Figure 3: Instantaneous multi-scale streamlines of a cylinder wake in the $(x, y)$-plane.

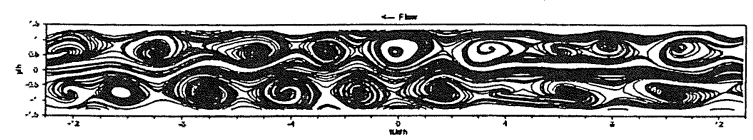

(a) $f=130 \mathrm{~Hz}$

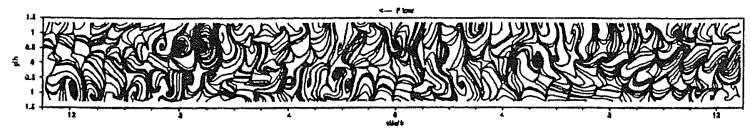

(b) $f=260 \mathrm{~Hz}$

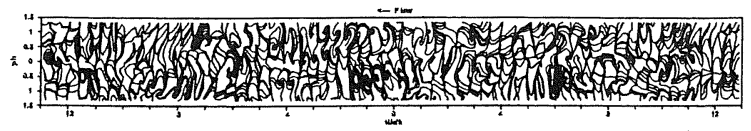

(c) $f=510 \mathrm{~Hz}$

Figure 4: Instantaneous multi-scale streamlines of a screen wake in the $(x, y)$-plane.

screen wake exhibit the elliptical shape. These differences reflect basic differences in the vortex formation mechanisms. Vortices in the circular cylinder wake are shed from the body surface, where those in the screen wake are likely to arise from instability in the developing mean wake profile.

The streamlines in Fig.3 (b), (c) and Fig.4 (b) show a number of structures that are smaller than those in Fig. 3 (a) and Fig.4 (a). Some of them are the small vortices that are contained in vortical structures. Others

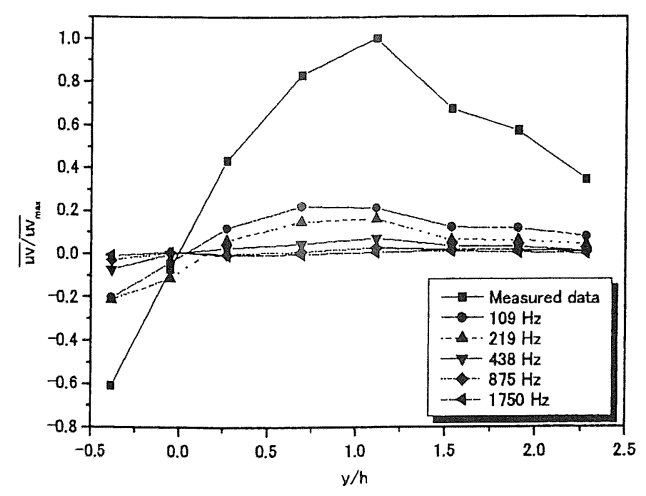

(a) Circular cylinder

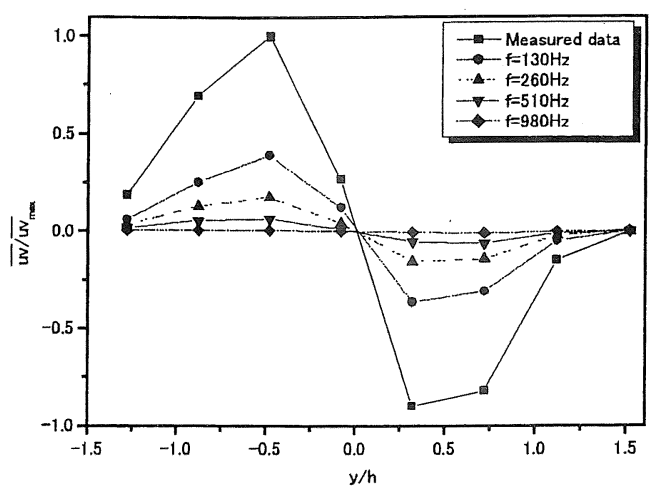

(b) Screen

Figure 5: Shear stress of various scales.

correspond to the saddle regions between the vortices. It is evident that the intermediate-scale structures of screen wake exhibit periodical and their size are almost uniform. However, the structures of cylinder wake exhibit irregular shapes and various sizes. As the central frequency increases, as shown in Fig.3 (d) and Fig.4(c), a number of even smaller scale structures are identifiable. Their occurrence is rather periodical, and the structures of screen wake are similar to that of cylinder wake.

\section{Reynolds Stresses of Various Scales}

The lateral distributions of time-averaged shear stress components at different central frequency for the cylinder and screen wakes, which are normalized by the maximum value of measured shear stress $\overline{u v}_{\max }$, are shown in Fig.5. The distributions of $\overline{u v} / \overline{u v}$ max component at each frequency are similar to that of the measured data for both of cylinder and screen wakes. The $\overline{u v} / \overline{u v}_{\max }$ component decreases when increasing frequency, and varies greatly with the central frequency. For the cylinder wake, as shown in Fig.5 (a), $\overline{u v} / \overline{u v}_{\max }$ component decreases rapidly with increasing the frequency in the range of high frequency $(f \geq 438 \mathrm{~Hz})$. The $\overline{u v} / \overline{u v}_{\max }$ component of $109 \mathrm{~Hz}$ remains everywhere largest in all components. The 


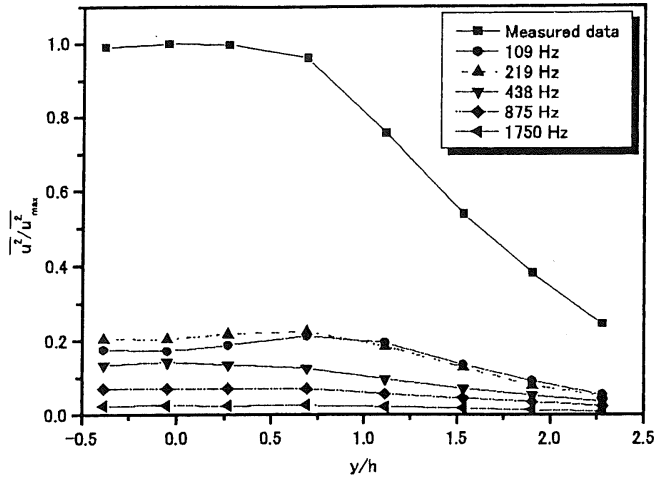

(a) Circular cylinder

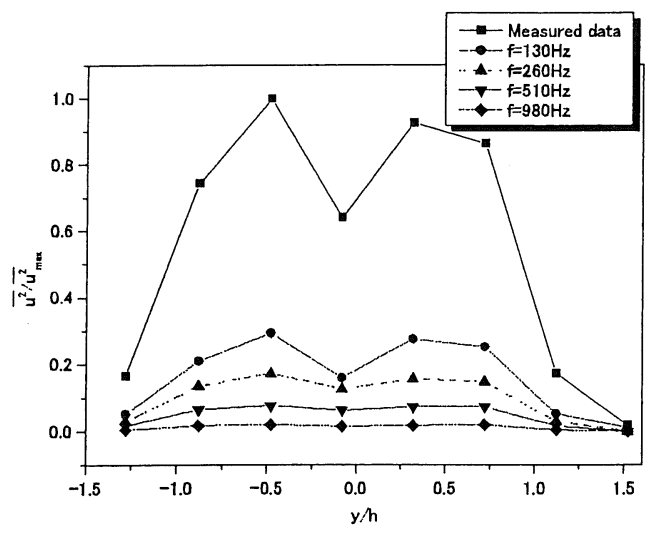

(b) Screen

Figure 6: Normal stress of various scales.

$\overline{u v} / \overline{u v}_{\max }$ component of $219 \mathrm{~Hz}$ is also evidently larger than other components of $f \geq 438 \mathrm{~Hz}$. This indicates that the contribution to the total shear stresses is greater from the large- and intermediate-scale structures. The peaks of $u v$ components at 109 and $219 \mathrm{~Hz}$ appear around $y / h=1.0$, which correspond to the averaged position of the vortex and saddle-point or rib-like structure. For the screen wake, $\overline{u v} / \overline{u v}_{\max }$ component of $130 \mathrm{~Hz}$ remains everywhere largest in all components. Peaks of $\overline{u v} / \overline{u v}_{\max }$ components at 130 $\mathrm{Hz}$ can be observed around $y / h=0.3$. The maximum contribution to total $\overline{u v}$ is about $35 \%$ from the component of $130 \mathrm{~Hz}$.

The distributions of time-averaged normal stress components $\overline{u^{2}} / \bar{u}^{2}$ max at various frequency for the cylinder and screen wakes are plotted in Fig.6. The distribution of $\overline{u^{2}} / \overline{u^{2}}{ }_{\max }$ component at each frequency is similar to that of the total normal stress. In the case of the cylinder wakes, $\overline{u^{2}} / \bar{u}^{2}$ max component decreases when increasing the central frequency. However $\overline{u^{2}} / \overline{u^{2}}$ max component of 219 $\mathrm{Hz}$ is higher than that of $109 \mathrm{~Hz}$ in the range of $y / d<$
0.7. This implies that the contribution to $\overline{u^{2}} / \overline{u^{2}} \max$ from the intermediate-scale structures is greater than that from large-scale structures due to the occurrence of the rib-like structures. For the screen wake, the component of $\overline{u^{2}} / \overline{u^{2}}$ max around the average frequency of $130 \mathrm{~Hz}$ shows largest value. This indicates that the greatest contribution to the total normal stress comes from the component of large-scale structure in the screen wake.

\section{Conclusions}

(1) The behavior of large- and intermediate-scale structures in wakes depends on the initial conditions. However, the behavior of small-scale structures is independent of the initial conditions.

(2) The most significant contribution to Reynolds stress and turbulent energy appears to come from the large-scale and rib-like structures in the cylinder wake. In the screen wake, however, only the large-scale structure exhibits more contribution.

\section{References}

Li, H., 1998, "Identification of Coherent Structure in Turbulent Shear Flow with Wavelet Correlation Analysis", ASME Journal of Fluids Engineering, Vol. 120, pp.778-785.

Li, H., Takei, M., Ochi, M., Saito, Y., and Horii, K, 1999, "Application of Two-dimensional Orthogonal Wavelets to Multi-resolution Image Analysis of a Turbulent Jet", Transactions of the Japan Society for Aeronautical and Space Sciences, Vol. 42, pp.120-127.

Sreenivasan, K. R., 1981, "Approach to Self-Preservation in Plane Turbulent Wakes", AIAA Journal, Vol.19, pp.1365-1367.

Zhou, Y., and Antonia, R.A., 1992, "Convection Velocity Measurements in a Cylinder Wake", Experiments in Fluids, Vol. 13, pp.63-70.

Zhou, Y., and Antonia, R.A., 1994, "Effect of Initial Conditions on Vortices in a Turbulent Near Wake", ALAA Journal, Vol. 32, pp.1207-1213. 